\title{
Effect of poly(thiophene)s topology on their third-order nonlinear optical response
}

Stien Vertommen ${ }^{\mathrm{a}}$, Olivier Deschaume ${ }^{\mathrm{b}}$, Carmen Bartic ${ }^{\mathrm{b}}$, Julien De Winter ${ }^{\mathrm{c}}$, Pascal Gerbaux $^{\mathrm{c}}$, Thierry Verbiest ${ }^{\mathrm{d}}$, Guy Koeckelberghs ${ }^{{ }^{*}}$ ${ }^{a}$ Department of Chemistry, Laboratory for Polymer Synthesis, KU Leuven, Celestijnenlaan 200F, box 2404, 3001 Heverlee, Belgium.

bDepartment of Physics and Astronomy, Soft-Matter Physics and Biophysics Section, KU Leuven, Celestijnenlaan 200D, box 2416, 3001 Heverlee, Belgium

'Organic Synthesis and Mass Spectrometry Laboratory, Interdisciplinary Center for Mass Spectrometry, University of Mons-UMons, 23 Place du Parc, 7000 Mons, Belgium

${ }^{\mathrm{d}}$ Department of Chemistry, Molecular Imaging and Photonics, KU Leuven, Celestijnenlaan 200D, box 2425, 3001 Heverlee, Belgium

*Email address: guy.koeckelberghs@kuleuven.be

\section{Abstract}

The research area of nonlinear topologies of conjugated polymers (CPs) became a topic of growing interest due to their unique optoelectronic properties compared to CPs with linear topologies. Nonetheless, the field of the third-order nonlinear optical response (TONO) is still largely unexplored, although, theoretically, nonlinear CPs should give a higher TONO response. Therefore, linear CPs and a trigonal nonlinear CP were synthesized via a combination of a controlled chain-growth polymerization, postpolymerization reactions and a click reaction. Afterward, the TONO response of the CPs was investigated with the highly sensitive third-harmonic scattering technique using solvatochromism experiments. In contrast to what was expected, trigonal nonlinear CPs do not possess a higher TONO response compared to their linear counterparts.

Keywords: Conjugated polymers, Kumada catalyst transfer condensative polymerization, third-order nonlinear optical response 


\section{Introduction}

During the last decades, CPs have gained a great deal of attention as a potential source for organic optoelectronics. ${ }^{1-7}$ Due to their unique optical and electronic properties, their easy processability and the possibility to control and design their molecular structure, CPs are ideal candidates to be used in optoelectronic applications. Even after decades of research, poly(thiophene)s (PTs) remain the benchmark CPs as a consequence of their impressive properties. Because of their effective conjugation length, synthetic versatility and stability, the study of the optoelectronic properties of PTs is still central to the research on CPs. ${ }^{8,918-20,10-17}$ The numerous studies already accomplished, demonstrate that most of the research is performed on linear CPs. However, the morphology of the polymer is important as well and nonlinear CPs can have a positive influence on the optoelectronic properties, which can offer a whole new range of opportunities for applications. ${ }^{21-28}$ Recently, the research on nonlinear CPs became a topic of growing current interest. Nonetheless, one specific important area is still largely unexplored. The research on the influence of a nonlinear topology on the third-order nonlinear optical response (TONO) response of CPs is uncharted, although theoretically, a nonlinear topology offers a lot of advantages. CPs -and certainly PTspossess an extended $\pi$-system with a high density of very polarizable $\pi$-electrons which features inherently a large TONO response. ${ }^{29,30}$ Besides, the TONO response intrinsically relies on the molecular second hyperpolarizability $\gamma$, which is a fourth rank tensor with 81 tensor components. ${ }^{31}$ The number of non-zero tensor components determines the TONO response and depends, among other things, on the topology. Linear CPs only have a contribution from polarizable $\pi$-electrons in one dimension along their conjugated polymer backbone. In contrast, nonlinear CPs possess contributions in two or even three dimensions, leading to more non-zero tensor components which would theoretically imply a higher $\gamma$ and hence a larger TONO response. In this regard, research on the TONO response of nonlinear CPs can offer a

60 material with a large TONO response, suitable for application in optical network components allowing for fast communication and larger bandwidths. ${ }^{32-36}$ These materials are in high demand in the telecommunication industry, but yet not 
straightforward due to an unoptimized TONO response. The incorporation of the optimal topology is a first step in realizing a breakthrough.

In order to investigate the influence of the topology on the TONO response, three different CPs were synthesized: two linear CPs (P1 and P2) and one nonlinear CP with a trigonal topology (P3). By combining a controlled chain-growth polymerization with post-polymerization reactions and an efficient click-reaction, maximal control over the polymer structure was obtained. An overview of the synthesized polymers is represented in Chart 1. The linear polymer P1 only contains the azide endfunctionalized polymer arm, while the linear polymer P2 and the trigonal nonlinear polymer $\mathbf{P 3}$ contain end-functionalized polymer arms coupled to a central core via the Copper(I)-catalyzed Azide Alkyne Cycloaddition ( $\mathrm{Cu}(\mathrm{I}) \mathrm{AAC})$ click reaction. Note that $\mathbf{P 2}$ forms an intermediate between $\mathbf{P 1}$ and P3. P2 possesses a linear topology like P1, but just as P3, it contains end-functionalized polymer arms coupled to a central core. In addition $\mathbf{P 1}$ is used as a reference polymer since both $\mathbf{P 2}$ and $\mathbf{P 3}$ are built from this polymer. By varying the amount of end-functionalized polymer arms, the topology can be altered from linear (P2) to trigonal (P3). Since the presence of the central core, the methylene and the triazole unit interrupts the conjugated backbone in the polymer structure of $\mathbf{P 2}$ and P3, the same conjugation length is maintained in P1-P3. Therefore, the influence of the conjugation length on the TONO response is excluded and differences in TONO response exclusively originate from the topology present in the polymer structure.

Finally, the TONO response of the CPs was measured in solution using the thirdharmonic scattering (THS) technique. ${ }^{37,38}$ This technique is very convenient and allows for an efficient, sensitive and fast measurement of the TONO response, leading to very trustworthy results. 
P1
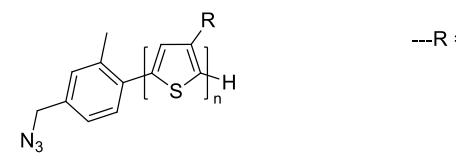

P2

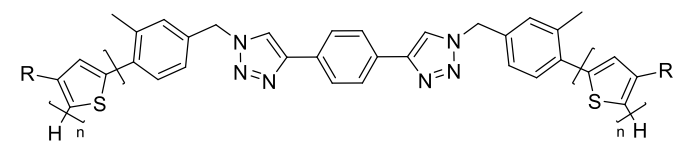

P3

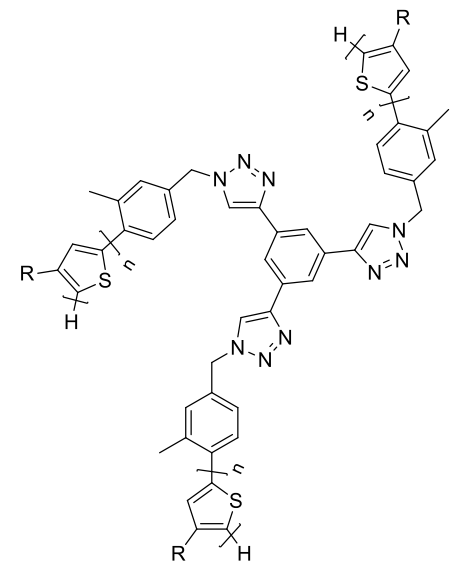

Chart 1: Overview of the synthesized polymers P1-P3.

\section{Results and discussion}

In order to synthesize P1-P3, three main synthetic steps were performed: (i) a controlled chain-growth polymerization combined with (ii) post-polymerization reactions to prepare $\mathbf{P 1}$ and finally (iii) a click reaction to synthesize $\mathbf{P 2}$ and $\mathbf{P 3}$, where azide end-functionalized polymer arms (P1) were coupled to a central core containing alkyne functions. This strategy is the "coupling-onto" method and is preferred over the "core-first" strategy and the "arm-first" strategy since the "coupling-onto" method is the most convenient strategy when a controlled polymerization is desired. ${ }^{25}$ An overview of the synthetic pathway is represented in Scheme 1. The precursor polymer P1a was synthesized using an externally initiated Ni-mediated Kumada catalyst transfer condensative polymerization (KCTCP).$^{39,40}$ The external functionalized initiator $\mathbf{1}$, which was responsible for implementing the functionalities on the polymer, underwent 
in situ a ligand exchange with 1,3-bis(diphenylphosphino)propane (dppp) in order to obtain initiator 2. Prior to polymerization, precursor monomer $\mathbf{3}$ was converted into monomer 4 using a Grignard metathesis reaction. The polymerization was terminated with acidified tetrahydrofuran whereafter precursor polymer P1a was obtained. Subsequently, two post-polymerization steps were performed, since it was not possible to introduce the azide function directly on the external initiator due to safety and incompatibility reasons. ${ }^{25}$ In a first reaction, the silyl-protected end group on the initiator was transformed to an alcohol group using an excess of tetrabutylammonium fluoride trihydrate $\left(\mathrm{TBAF} \cdot 3 \mathrm{H}_{2} \mathrm{O}\right)$ to achieve $\mathbf{P 1 b}$. The azide end-functionalized polymer arm P1 was obtained in a second reaction where the alcohol group was converted to an azide function using diphenylphosphoryl azide (DPPA) and 1,8diazabicycloundec-7-ene (DBU).
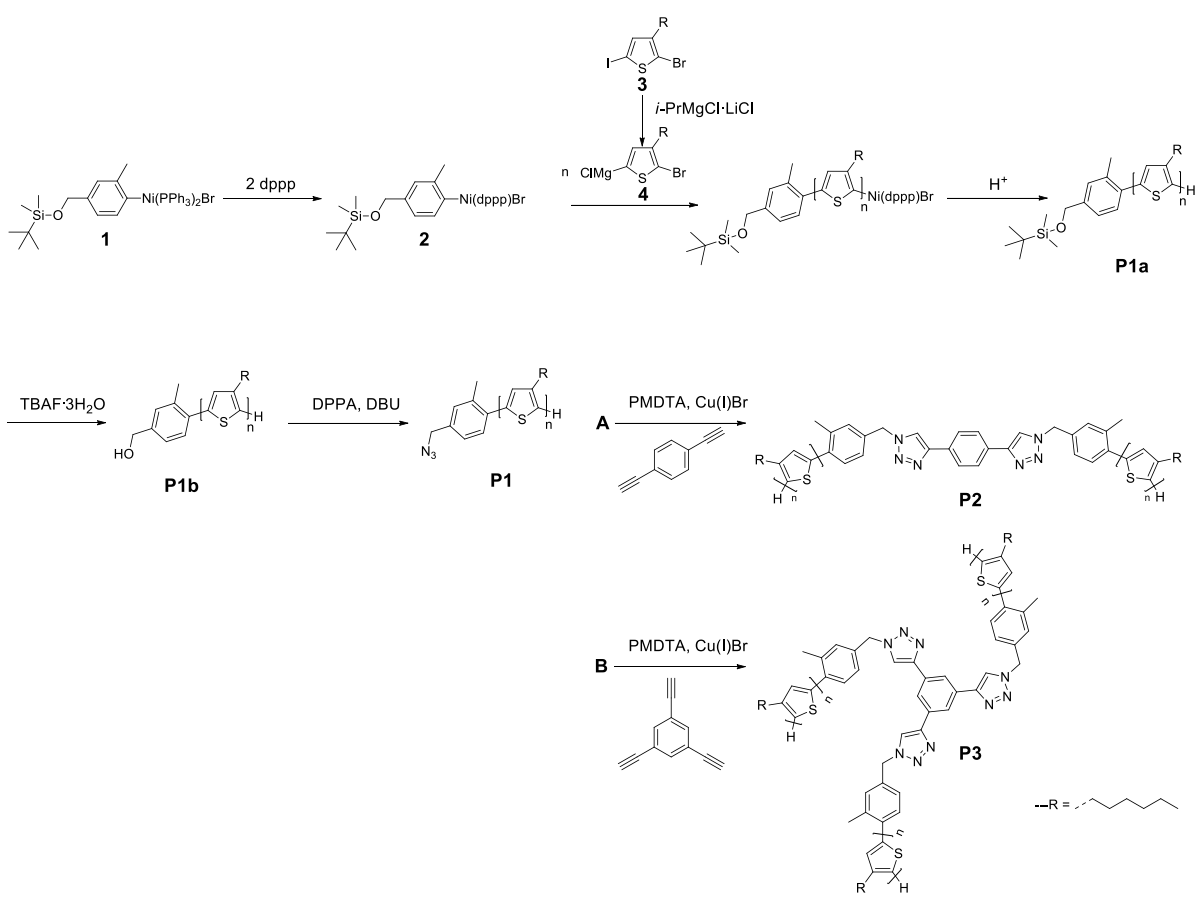

Scheme 1: Representation of the synthetic pathway to obtain P1-P3.

After every polymerization and post-polymerization step, the controlled character of KCTCP and the quantitative conversion, respectively, was verified by a combination of gel permeation chromatography (GPC) and proton nuclear magnetic resonance $\left({ }^{1} \mathrm{H}\right.$ 
NMR). GPC measures the number average molar mass $\left(\overline{\boldsymbol{M}}_{\boldsymbol{n}}\right)$ and the molar mass dispersity $\left(\bigoplus_{M}\right)$, while the degree of polymerization (DP) was estimated using ${ }^{1} \mathrm{H}$ NMR. An overview of the obtained results is represented in Table 1. According to Figure 1, the DP was obtained by determining the ratio between the integrated signal of the $\alpha$ methylene protons of the thiophene units over the integrated signal of the methyl protons of the external initiator. In addition, ${ }^{1} \mathrm{H}$ NMR was used as well to monitor the conversion of the post-polymerization reactions. The ${ }^{1} \mathrm{H}$ NMR signal of the $\mathrm{CH}_{2}$-group next to the functional group of the initiator shifts depending on the functional group. ${ }^{25}$ Therefore, the post-polymerization reactions were monitored with ${ }^{1} \mathrm{H}$ NMR until full conversion was reached. Finally, the ${ }^{1} \mathrm{H}$ NMR spectra reveal that the polymers are $\mathrm{H}$ terminated. ${ }^{41}$ The triplet at $2.6 \mathrm{ppm}$ indicates an H-terminated polymer chain. Since no other peaks are present in this region and since the ratio of 3 protons for the methyl protons of the initiator and 2 protons for the $\alpha$-methylene protons of the last thiophene unit was present, a controlled polymerization was obtained with optimal control over the polymer structure. In order to get one more confirmation about the polymer structures, MALDI-ToF measurements were performed. Figure 2 reports the mass spectrum recorded for $\mathbf{P 1}$. As expected, one main distribution is present and corresponds to $\mathbf{P 1}$ H-terminated in one side and the desired functional end groups at the other side. Note that a small subset of peaks at low molar mass (centered around 11) seems to be present. The masses of the MALDI-ToF spectrum of the subset corresponds to a P3HT with the same end-groups as the main set and the separation between the peaks corresponds with the mass of one repeating unit. This shows that the nature of the polymers of both sets are the same, but that the subset has a lower molar mass. Note that MALDI-ToF overestimates lower molar masses. On GPC, only one set is visible. So, either it concerns a very minor fraction that is not visible by GPC and negligible, or it is simply due to the MALDI process. In the supporting information (SI) a comparison with the theoretical model is represented in Figure S9. The corresponding GPC chromatograms (Figure S1-S3) and ${ }^{1} \mathrm{H}$ NMR spectra (Figure S4-S6) are found in the SI. 


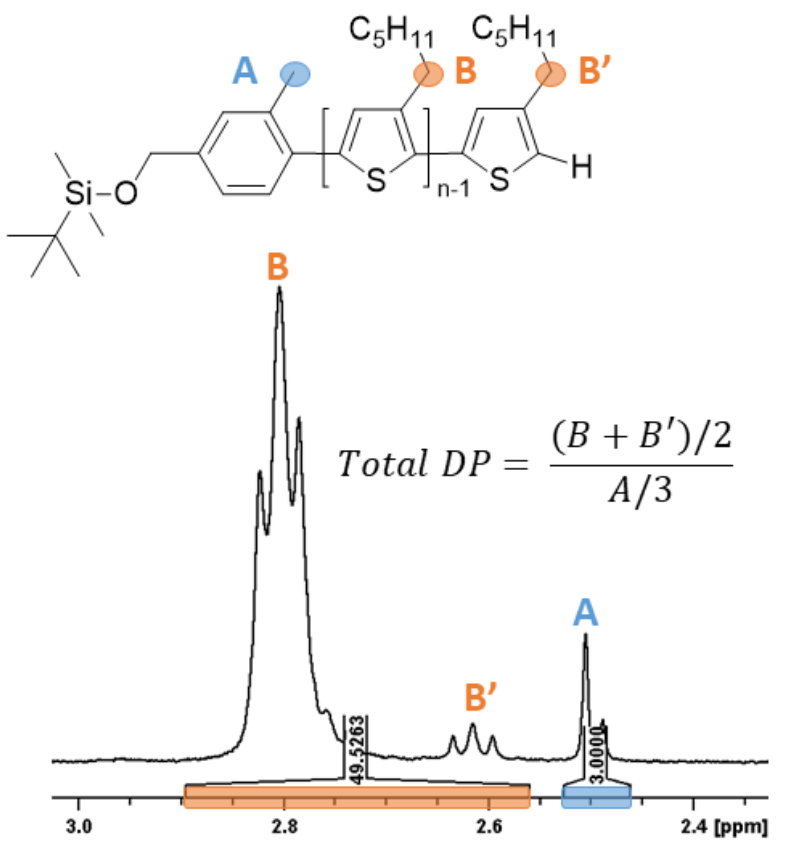

Figure 1: Determination of DP using ${ }^{1} \mathrm{H}$ NMR.

Table 1: Overview of the GPC results $\left(\bar{M}_{n}\right.$ and $\left.\Xi_{M}\right)$ and ${ }^{1} H$ NMR results (DP) for P1a-P1.

\begin{tabular}{cccc}
\hline & $\overline{\boldsymbol{M}}_{\boldsymbol{n}} \mathbf{( k g / \mathbf { m o l } )}$ & $\boldsymbol{Ð}_{\mathbf{M}}$ & DP \\
\hline P1a & 5.58 & 1.2 & 25 \\
\hline P1b & 5.60 & 1.2 & 25 \\
\hline P1 & 6.03 & 1.2 & 25 \\
\hline
\end{tabular}




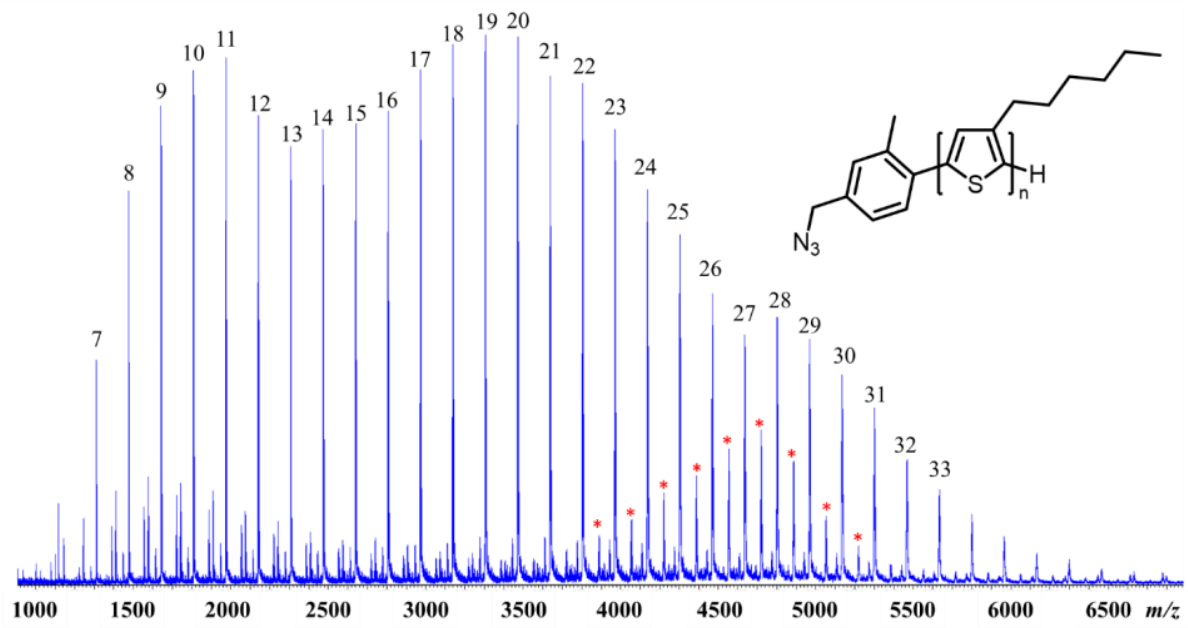

Figure 2: MALDI-ToF spectra of P1. The number on the top of each signal corresponds to the number of monomer units, * corresponds to adducts of the polymer with matrix molecules (DCTB).

To synthesize P2 and P3, the azide end-functionalized polymer arm (P1) was coupled to a central core containing alkyne functions using the $\mathrm{Cu}(\mathrm{I}) \mathrm{AAC}$ click reaction with $\mathrm{Cu}(\mathrm{I}) \mathrm{Br}$ as catalyst and $\mathrm{N}, \mathrm{N}, \mathrm{N}^{\prime}, \mathrm{N}^{\prime \prime}, \mathrm{N}^{\prime \prime}$-pentamethyldiethylenetriamine (PMDTA) as ligand. For P2, synthetic route A (Scheme 1) was followed, while synthetic route B (Scheme 1) was followed for P3. In both cases, an excess of azide end-functionalized polymer arm P1 was used with respect to the central core to ensure complete conversion. Therefore, after completion of the $\mathrm{Cu}(\mathrm{I}) \mathrm{AAC}$ click reaction, both polymers were purified by preparative GPC in order to remove the non-reacted azide end-functionalized polymers. The obtained GPC chromatograms before and after preparative GPC are represented in Figure 3. 

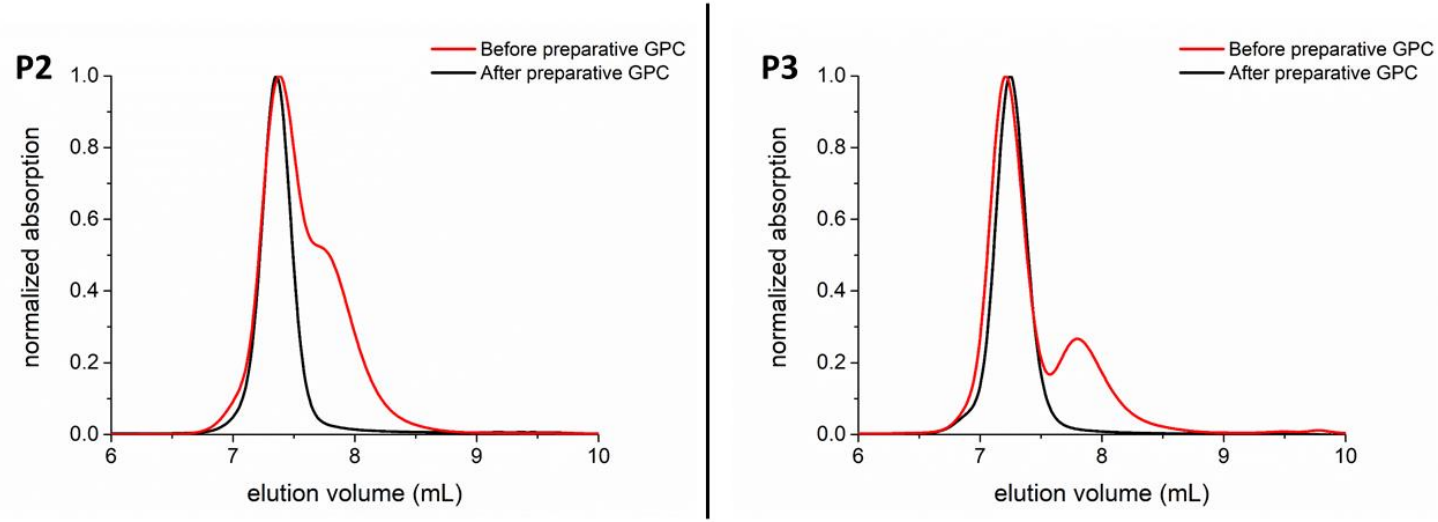

Figure 3: GPC chromatograms of P2 and P3 before and after preparative GPC

After purification, GPC analysis was performed to estimate the $\overline{\boldsymbol{M}}_{\boldsymbol{n}}$, the $\bigoplus_{\mathrm{M}}$ and ${ }^{1} \mathrm{H}$ NMR was measured to indicate that the click reaction and the removal of the free polymer were successful. ${ }^{25}$ The ${ }^{1} \mathrm{H}$ NMR spectra of $\mathbf{P 2}$ and $\mathbf{P 3}$ are found in the SI (Figure S7-S8), while the obtained GPC results are represented in Table 2. Note that the $\overline{\boldsymbol{M}}_{\boldsymbol{n}}$ is approximately two (for $\mathbf{P 2}$ ) and three (for P3) times higher compared to P1, indicating the success of the click reaction as well. ${ }^{1} \mathrm{H}$ NMR reveals a DP of 32 and 27 for $\mathbf{P 2}$ and P3, respectively. Furthermore, MALDI-ToF measurements were performed for $\mathbf{P} 2$ and $\mathbf{P 3}$ confirming that the expected structure was obtained. The spectra were represented in Figure 4A (for P2) and Figure 4B (for P3). Comparison with the theoretical model is presented in the SI (Figure S10-S11).

Table 2: The obtained GPC results of $P 2$ and $P 3$

\begin{tabular}{ccc}
\hline & $\overline{\boldsymbol{M}}_{\boldsymbol{n}}(\mathrm{kg} / \mathrm{mol})$ & $\boldsymbol{Ð}_{\mathrm{M}}$ \\
\hline P2 & 14.7 & 1.05 \\
\hline P3 & 17.6 & 1.06 \\
\hline
\end{tabular}



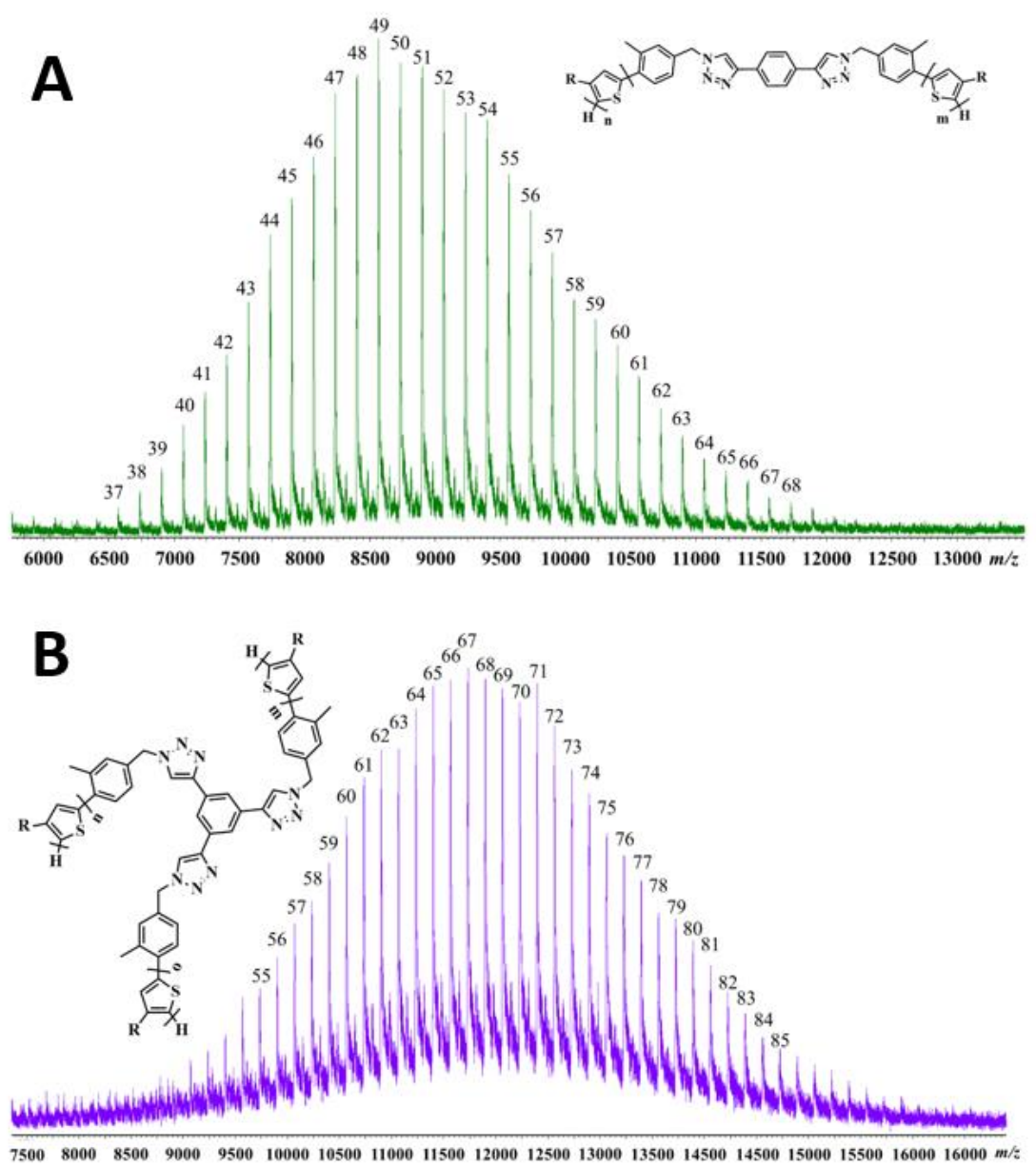

Figure 4: A: MALDI-ToF spectra of P2. The number on the top of each signal corresponds to the total number of monomer units $(n+m)$. B : MALDI-ToF spectra of $P 3$. The number on the top of each signal corresponds to the total number of monomer units $(\mathbf{n}+\mathbf{m}+\mathbf{o})$.

After the successful synthesis of P1-P3, UV-vis spectroscopy and THS measurements were conducted by performing solvatochromism experiments. CPs dissolved in a good solvent, chloroform, behave like a random coil, which means that they are not in their extended conjugated state. As a consequence, no THS signal is detected. ${ }^{38}$ Upon the incremental addition of nonsolvent, methanol, under continuous stirring and at a constant speed, supramolecular organization is induced in the CPs. This is evidenced by the appearance of a THS signal due to an increase in effective 
conjugation length resulting from a planarization and upcoming $\pi-\pi$ interactions, and by the appearance of a redshift and a vibronic finestructure in the UV-vis spectrum. Since supramolecular organization is required to measure a THS signal, the obtained TONO response originates from the polymer aggregates. The molecular response of the polymer aggregates can be obtained by measuring the second hyperpolarizability $\gamma$ using the THS technique. ${ }^{37,38,42,43}$

UV-vis spectroscopy was conducted to confirm that the same conjugation length is maintained for P1-P3. P2 and P3 contain more polymer arms attached to a central core, but since the central core, the methylene and the triazole unit interrupt the conjugated backbone, the conjugation length should be the same. The obtained UV-vis spectra are represented in Figure 5. This reveals that $\lambda_{\max }$ in chloroform is within experimental error similar, showing that the conjugation length is indeed not affected. However, an intriguing trend is visible in the extinction coefficient, as it increases from P1 to P3. The vibronic peaks resulting from $\pi-\pi$ interactions ( $548 \mathrm{~nm}$ and $600 \mathrm{~nm}$ ) are situated at the same wavelength for P1-P3, indicating that the conjugation length is maintained, meaning there would be no influence of the conjugation length on the TONO response. Note that P1-P3 differ in the amount of $\pi-\pi$ interactions in their polymer aggregates. The Spano model is used to evaluate the strength of the $\pi-\pi$ interactions present, determined by the ratio between the line strength of the $0-0$ transition over the $0-1$ transition in the absorption spectrum. ${ }^{44,45}$ The deconvolution of the UV-vis spectra and the related calculations are found in the SI (Figure S12-S14). The deconvolution of the UV-vis spectra was performed at the percentage of methanol just before precipitation took place (50\% of methanol for P1; $33 \%$ of methanol for $\mathbf{P 2}$ and $29 \%$ of methanol for P3). The obtained ratio for $\mathbf{P 1}$ is $2.82 \pm 0.74$, while the strength of $\pi-\pi$ interactions for $\mathbf{P 2}$ and $\mathbf{P 3}$ are respectively $4.34 \pm 0.61$ and $4.14 \pm 0.19$. This indicates enhanced $\pi-\pi$ interactions and hence a stronger tendency to aggregate in contrast to $\mathbf{P 1}$. This is in line with previous findings where star-shaped polymers show a stronger supramolecular organization compared to single polymer arms. ${ }^{25}$ 


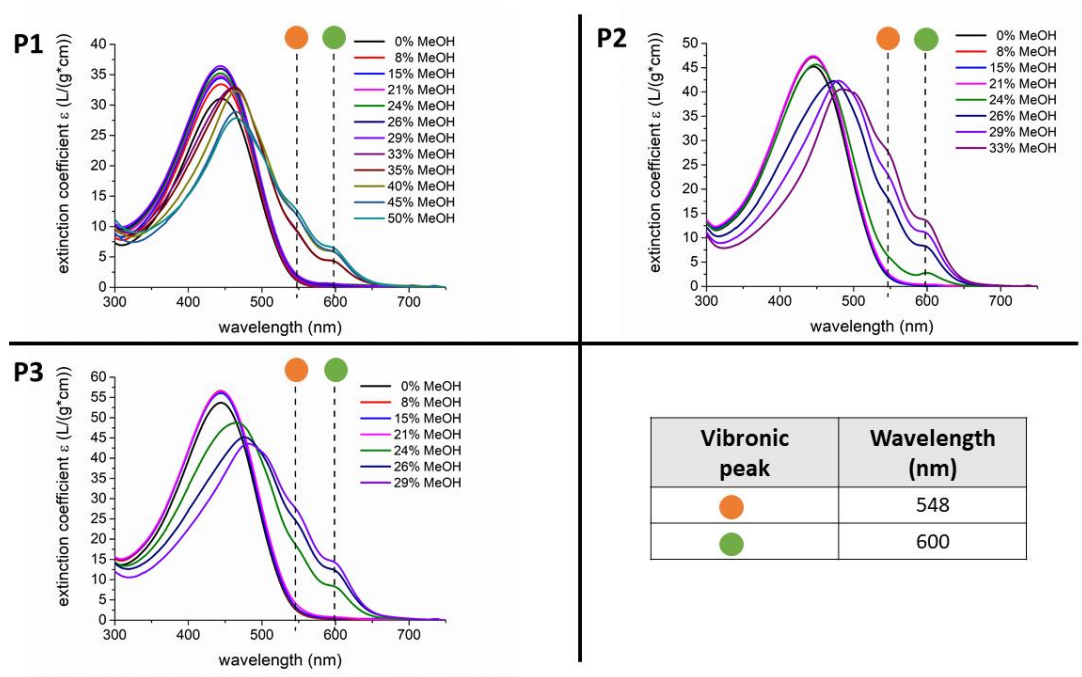

Figure 5: UV-vis spectra of P1-P3. The different vibronic peaks are marked.

THS, which is a non-invasive and sensitive nonlinear scattering technique, was used to evaluate the TONO response. The polymer solution was illuminated with high intensity polarized light $\mathrm{I}_{\omega}$, originating from a high-power pulsed laser beam at an incident wavelength of $1300 \mathrm{~nm}$, which is the most favorable excitation laser wavelength for conjugated polymers. ${ }^{46}$ Since third-order nonlinear scattering implies that scattering of the output photons occurs at the triple frequency $3 \omega$ compared to the incident frequency $\omega$, the intensity of the scattered light $\mathrm{I}_{3 \omega}$ that contributes to the THS signal is detected at one-third of the incident wavelength, implying detection at 433 $\mathrm{nm} .{ }^{42,43} \mathrm{In}$ addition, the measured intensity $\mathrm{I}_{3 \omega}$ originates completely from the polymer aggregates, since the signal coming from the solvents, chloroform and methanol, is negligible. ${ }^{38}$

At first, THS measurements were performed using a static solvatochromism approach. Methanol was added stepwise to the polymer solution under continuous stirring $(500 \mathrm{rpm})$ and at a constant speed $(0.25 \mathrm{~mL} / \mathrm{min})$ using an automatic syringe pump. THS spectra were recorded after each methanol addition. The obtained spectra are represented in Figure 6. The THS signal, in which we are interested, is the small peak located at $433 \mathrm{~nm}$. The broad band located at longer wavelengths corresponds to multiphoton fluorescence and the small peak at $650 \mathrm{~nm}$ originates from the second harmonic scattering signal. 


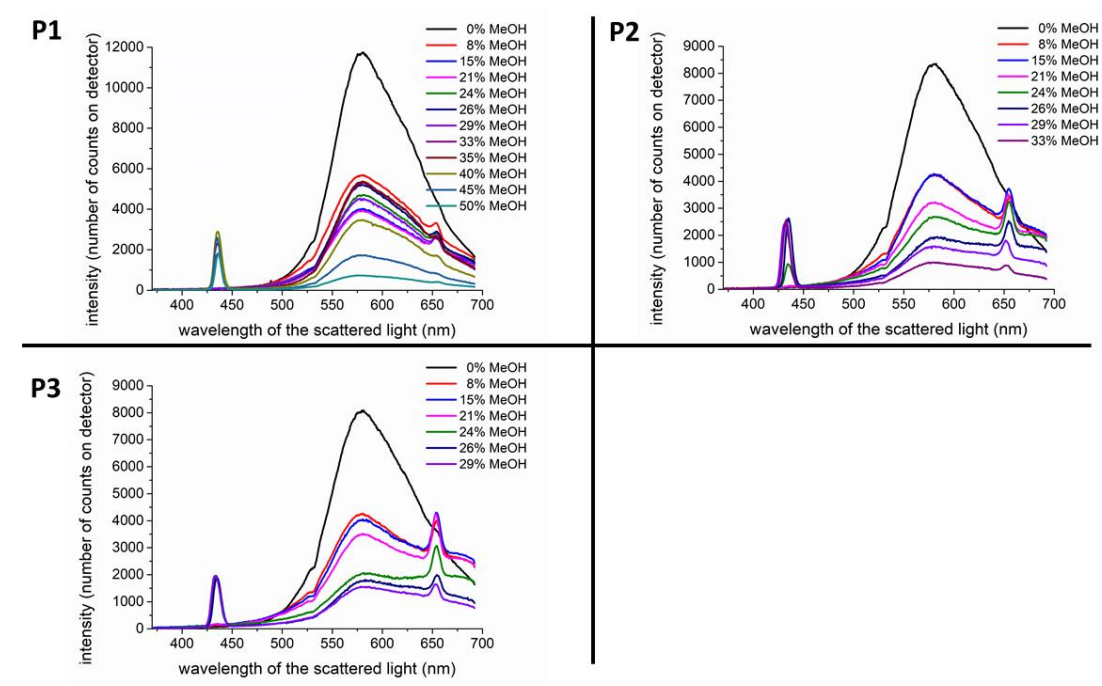

Figure 6: THS spectra of P1-P3 according to the static solvatochromism experiments. The THS signal, in which we are interested, is the small peak located at $433 \mathrm{~nm}$. The broad band located at longer wavelengths corresponds to multiphoton fluorescence and the small peak at $650 \mathrm{~nm}$ originates from the second harmonic scattering signal.

The obtained intensity $\mathrm{I}_{3 \omega}$ of the TONO response for P1-P3 is situated around the same number of counts on the detector (between 2000 and 3000 counts), with a slightly lower detectable response for $\mathbf{P 3}$, meaning that there is only a small influence of the topology on the TONO response of the polymer aggregates. Although P3 shows a slightly lower TONO response, the difference is not significant. In order to refine the observations, another THS measurement was conducted. Again, a solvatochromism experiment was performed, but in contrast to the previous measurement where the nonsolvent was added stepwise, methanol was continuously added at a constant speed $(0.07 \mathrm{~mL} / \mathrm{min})$ until a percentage of $60 \%$ methanol was reached, while the polymer solution, which was placed in the THS set-up, was stirred and THS spectra were recorded simultaneously. The intensity $\mathrm{I}_{3 \omega}$ was recorded continuously as a function of the percentage of methanol added, whereafter the area of the THS peak $\mathrm{A}_{\mathrm{THS}}$ was determined using a Gaussian fit function. The concentration corrected obtained area $\mathrm{A}_{\mathrm{THS}}$ was plotted against the percentage of methanol added and the resulting plot is represented in Figure 7. 


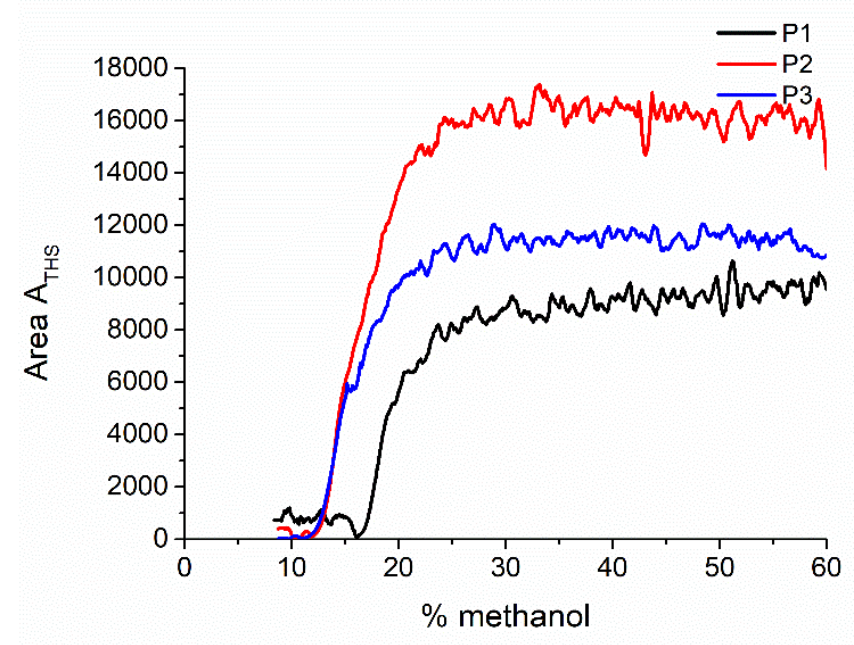

Figure 7: Area Atrs as a function of the percentage of methanol added for P1P3.

The obtained area under the THS peak, which is a measure for the magnitude of the TONO response, is almost twice as high for $\mathbf{P 2}$ compared to $\mathbf{P 1}$. This difference is attributed to the strength of $\pi-\pi$ interactions since both polymers have a linear topology. According to the Spano model, $\mathbf{P} 2$ possesses stronger $\pi-\pi$ interactions. Since previous research demonstrated that excellent $\pi-\pi$ interactions give rise to a large TONO response, ${ }^{46}$ the larger TONO response of $\mathbf{P} 2$ compared to $\mathbf{P 1}$ can be explained by the stronger $\pi-\pi$ interactions present. However, since the strength of the $\pi-\pi$ interactions between $\mathbf{P 2}$ and $\mathbf{P} \mathbf{3}$ is the same, they cannot be responsible for the difference in TONO response, meaning that the topology is responsible as it is the only varying parameter between P2 and P3. Surprisingly, trigonal nonlinear topologies do not lead to a higher TONO response of the polymer aggregates compared to their corresponding linear counterparts.

As already been stated, CPs have to be in their supramolecular organized state to detect a THS signal, meaning that the measured TONO response originates from the polymer aggregates. In order to evaluate the TONO response at the molecular level of the CPs, the second hyperpolarizability $\gamma$ was calculated from the polymer aggregates using Equations (1) and (2): ${ }^{37,38}$

$$
<\gamma^{2}>\sim \frac{I_{3 \omega}}{N I_{\omega}^{3}}
$$




$$
<\gamma>\sim \sqrt{\frac{I_{3 \omega}}{N I_{\omega}^{3}}}
$$

Where the brackets denote oriental averaging over all possible molecular orientations, $I_{3 \omega}$ the scattered intensity at the triple frequency, $I_{\omega}$ the intensity of the incident wavelength and $\mathrm{N}$ the concentration of the polymer solution, where dilution with methanol was taken into account. The obtained $\langle\gamma\rangle$ was plotted in function of the added percentage of methanol and is represented in Figure 8.

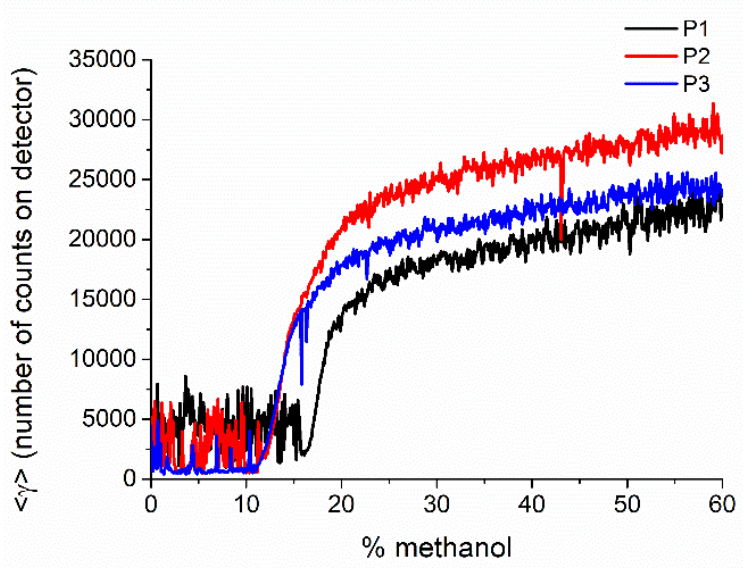

Figure 8: Obtained $<\gamma>$ as a function of the percentage methanol for P1-P3.

The same conclusion as from Figure 8 can be concluded, with the exception that the differences on the molecular level are smaller and the obtained $\langle\gamma\rangle$ lie closer to each other. Note that the obtained $\langle\gamma\rangle$ are in the same range with $\langle\gamma\rangle$ obtained from previous research on poly(thiophene)s. This confirms that poly(thiophene)s lead to a high TONO response. ${ }^{46}$ For the linear polymers, $\mathbf{P 1}$ and P2, P2 shows a higher $\langle\gamma\rangle$ as a result of enhanced $\pi-\pi$ interactions. While, when the trigonal nonlinear polymer is compared to its linear counterpart P2, the trigonal nonlinear topology does not give a higher $\langle\gamma\rangle$. Although in theory, nonlinear topologies should give rise to a higher TONO response, this effect is not experimentally verified. Modifying a linear topology to a trigonal nonlinear topology with the same amount of $\pi-\pi$ interactions does not lead to a significant enhancement of the TONO response. 
Finally, dynamic light scattering (DLS) measurements were performed to evaluate whether the TONO response was related to the size of the polymer aggregates. The size of the formed polymer aggregates was measured at several percentages of methanol added under solvatochromism experimental conditions. The diameter and the polydispersity index (PDI) are represented in Figure 9.
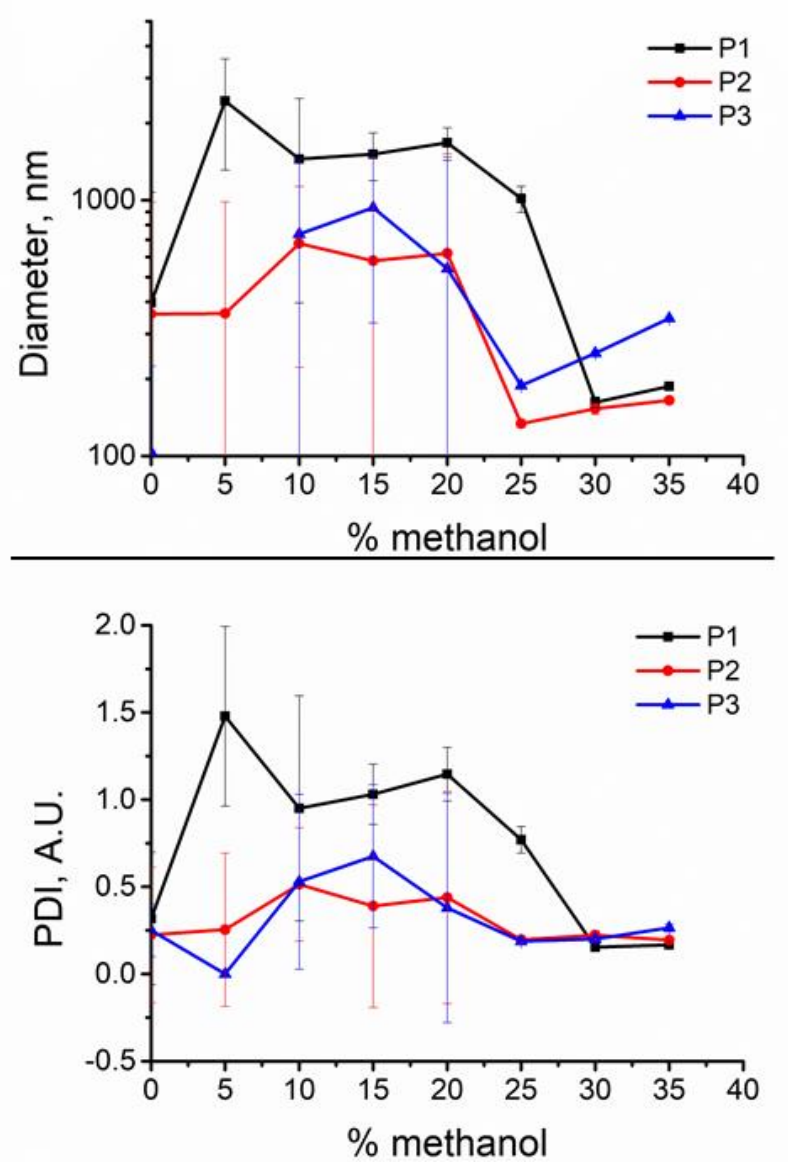

Figure 9: Diameter and PDI of the formed polymer aggregates for P1-P3.

Before the start of the supramolecular organization (the region between 0 and 20\% methanol for $\mathbf{P 2}$ and $\mathbf{P 3}$, the region between 0 and $25 \%$ methanol for $\mathbf{P 1}$ ), large diameter sizes are present as a result of the formation of solvent clusters. ${ }^{38}$ However, with the start of the supramolecular organization, the diameter decreases upon expelling the solvent out of the cluster and stacks of polymer aggregates are formed. Upon adding 
more methanol, the diameter of the polymer aggregates slightly increases with a larger detectable diameter for $\mathbf{P 3}$ compared to $\mathbf{P 2}$ and $\mathbf{P 1}$. The results of the DLS measurements are given up to $35 \%$ methanol, since flocculation occurred for higher methanol percentages. Although, trigonal nonlinear CPs maintain a larger hydrodynamic volume compared to their linear counterparts, the results cannot be correlated to the obtained TONO response and absorption spectra. The diameters observed for $\mathbf{P 1}$ and $\mathbf{P 2}$ are almost comparable, while the two polymers show a clear difference in vibrational finestructure in the $U V$-vis spectrum and a difference in TONO response. In contrast, $\mathbf{P} 2$ and $\mathbf{P} 3$ have the same amount of $\pi-\pi$ interactions, but the size of the polymer aggregates are slightly different and there is as well no apparent correlation with the TONO response for P1, P2 and P3.

It is clear that the trigonal topology does not increase the overall TONO response. One possible explanation could be that also a contribution of quadrupole interactions is present that lowers the overall TONO response. Indeed, a trigonal geometric leads to quadrupole interactions that result in a strong TONO response. Second, if the orientation of the arms towards each other is not random, cooperative and anticooperative effects can be present and the TONO response does not equal the sum of the individual arms. ${ }^{47}$ This can therefore explain the observed effects.

\section{Conclusions}

Two linear CPs and one trigonal nonlinear CP were successfully synthesized using $\mathrm{KCTCP}$, post-polymerization reactions and the $\mathrm{Cu}(\mathrm{I}) \mathrm{AAC}$ click reaction, whereafter they were measured with THS in order to evaluate the TONO response using solvatochromism experiments. The three CPs have the same conjugation length, so this parameter cannot explain differences in TONO response. The two linear CPs have a different amount of $\pi-\pi$ interactions, which can explain their different TONO response. However, since the trigonal nonlinear CP has the same strength of $\pi-\pi$ interactions as its linear counterpart, a difference in TONO response between these two polymers must be of topological origin. Although trigonal nonlinear topologies could have a higher TONO response compared to linear onces, as more contribution can be present, this 
effect was not experimentally found. The measured TONO response is lower compared to its linear counterpart with the same amount of $\pi-\pi$ interactions, meaning that trigonal nonlinear CPs do not enhance the TONO response. The difference in TONO response must therefore be attributed to cooperative anti-cooperative effects between conjugated systems that are electronically uncoupled and oriented towards each other by the coupling to a central core in a given geometry.

\title{
Conflicts of interest
}

"There are no conflicts to declare".

\begin{abstract}
Abbrevations
$\mathrm{CPs}$, conjugated polymers; $\mathrm{Cu}(\mathrm{I}) \mathrm{AAC}$, Copper(I)-catalyzed Azide Alkyne Cycloaddition; Đ, dispersity; DBU, 1,8-diazabicycloundec-7-ene; DP, degree of polymerization; DPPA, diphenylphosphoryl azide; dppp, 1,3365 bis(diphenylphosphino)propane; DLS, dynamic light scattering; $\gamma$, second hyperpolarizability; GPC, gel permeation chromatography; KCTCP, Kumada catalyst transfer condensative polymerization; $\overline{\boldsymbol{M}}_{\boldsymbol{n}}$, number average molar mass; PDI,

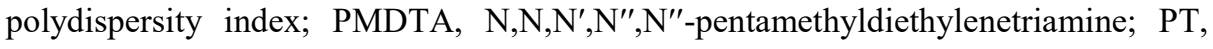
poly(thiophene); SI, supporting information; THS, third-harmonic scattering; TONO, 370 third-order nonlinear optical.
\end{abstract}

\section{Notes}

Supporting Information is available free of charge: Materials, Instrumentation, experimental details, Additional GPC chromatograms, ${ }^{1} \mathrm{H}$ NMR spectra, MALDI-ToF spectra, Deconvoluted UV-vis spectra. 


\section{Acknowledgements}

This research was funded by Onderzoeksfonds KU Leuven/ Research Fund KU Leuven (Research project G099319N) and Fund for Scientific Research (FWOVlaanderen-SB - 1SB5419N). S.V. is a doctoral fellow of the Fund for Scientific Research-SB (FWO-Vlaanderen). C.B. acknowledges the financial support of the Research Foundation-Flanders, Belgium (FWO research grant G0947.17N) and KU Leuven (research grant C14/18/061). O.D. acknowledges the financial support from KU Leuven (research grant C14/16/063). The MS lab is grateful to the "Fonds National de la Recherche Scientifique (FRS-FNRS)" for financial support for the acquisition of the Waters QTof Premier mass spectrometer.

\section{References}

1. Sirringhaus, H., Brown, P. J., Friend, R. H., Nielsen, M. M., Bechgaard, K., Langeveld-Voss, B. M. W., Spiering, A. J. H., Janssen, R. A. J., Meijer, E. W., Herwig, P. \& De Leeuw, D. M. Two-dimensional charge transport in conjugated polymers. Nature 401, 685-688 (1999).

2. Yan, H., Chen, Z., Zheng, Y., Newman, C., Quinn, J. R., Dötz, F., Kastler, M. \& Facchetti, A. A high-mobility electron-transporting polymer for printed transistors. Nature 457, 679-686 (2009).

3. Cheng, Y. J., Yang, S. H. \& Hsu, C. S. Synthesis of conjugated polymers for organic solar cell applications. Chem. Rev. 109, 5868-5923 (2009).

4. Klauk, H. Organic thin-film transistors. Chem. Soc. Rev. 39, 2643-2666 (2010).

5. Thompson, B. C. \& Fréchet, J. M. J. Polymer-fullerene composite solar cells. Angew. Chemie - Int. Ed. 47, 58-77 (2008).

6. Deibel, C. \& Dyakonov, V. Polymer-fullerene bulk heterojunction solar cells. Reports Prog. Phys. 73, (2010).

7. Sirringhaus, H., Kawase, T., Friend, R. H., Shimoda, T., Inbasekaran, M., Wu, W. \& Woo, E. P. High-resolution inkjet printing of all-polymer transistor circuits. Science 290, 2123-2126 (2000).

8. Smeets, A., Van Den Bergh, K., De Winter, J., Gerbaux, P., Verbiest, T. \& 
Koeckelberghs, G. Incorporation of different end groups in conjugated polymers using functional nickel initiators. Macromolecules 42, 7638-7641 (2009).

9. McDearmon, B., Lim, E., Lee, I. H., Kozycz, L. M., O’Hara, K., Robledo, P. I., Venkatesan, N. R., Chabinyc, M. L. \& Hawker, C. J. Effects of Side-Chain Topology on Aggregation of Conjugated Polymers. Macromolecules 51, 2580 2590 (2018).

10. Koppe, M., Brabec, C. J., Heiml, S., Schausberger, A., Duffy, W., Heeney, M. $\& \mathrm{McCulloch}$, I. Influence of molecular weight distribution on the gelation of $\mathrm{P} 3 \mathrm{HT}$ and its impact on the photovoltaic performance. Macromolecules 42, 4661-4666 (2009).

11. Goto, H., Okamoto, Y. \& Yashima, E. Solvent-induced chiroptical changes in supramolecular assemblies of an optically active, regioregular polythiophene. Macromolecules 35, 4590-4601 (2002).

12. Kohn, P., Huettner, S., Komber, H., Senkovskyy, V., Tkachov, R., Kiriy, A., Friend, R. H., Steiner, U., Huck, W. T. S., Sommer, J. U. \& Sommer, M. On the role of single regiodefects and polydispersity in regioregular poly(3hexylthiophene): Defect distribution, synthesis of defect-free chains, and a simple model for the determination of crystallinity. J. Am. Chem. Soc. 134, 4790-4805 (2012).

13. Tu, G., Li, H., Forster, M., Heiderhoff, R., Balk, L. J. \& Scherf, U. Conjugated triblock copolymers containing both electron-donor and electron-acceptor blocks. Macromolecules 39, 4327-4331 (2006).

14. Trznadel, M., Pron, A., Zagorska, M., Chrzaszcz, R. \& Pielichowski, J. Effect of molecular weight on spectroscopic and spectroelectrochemical properties of regioregular poly(3-hexylthiophene). Macromolecules 31, 5051-5058 (1998).

15. Kiriy, N., Jähne, E., Adler, H. J., Schneider, M., Kiriy, A., Gorodyska, G., Minko, S., Jehnichen, D., Simon, P., Fokin, A. A. \& Stamm, M. Onedimensional aggregation of regioregular polyalkylthiophenes. Nano Lett. 3, 707-712 (2003).

16. Van Den Eede, M. P., Bedi, A., Delabie, J., De Winter, J., Gerbaux, P. \& Koeckelberghs, G. The influence of the end-group on the chiral self-assembly 
of all-conjugated block copolymers. Polym. Chem. 8, 5666-5672 (2017).

17. Jaymand, M., Hatamzadeh, M. \& Omidi, Y. Modification of polythiophene by the incorporation of processable polymeric chains: Recent progress in synthesis and applications. Prog. Polym. Sci. 47, 26-69 (2015).

18. Verheyen, L., Leysen, P., Van Den Eede, M. P., Ceunen, W., Hardeman, T. \& Koeckelberghs, G. Advances in the controlled polymerization of conjugated polymers. Polymer 108, 521-546 (2017).

19. Willot, P., Steverlynck, J., Moerman, D., Leclère, P., Lazzaroni, R. \& Koeckelberghs, G. Poly(3-alkylthiophene) with tuneable regioregularity: Synthesis and self-assembling properties. Polym. Chem. 4, 2662-2671 (2013).

20. Verheyen, L., Timmermans, B. \& Koeckelberghs, G. The influence of branching on the Kumada catalyst transfer condensative polymerization of 3alkylthiophenes. Polym. Chem. 8, 2327-2333 (2017).

21. Yuan, M., Okamoto, K., Bronstein, H. A. \& Luscombe, C. K. Constructing regioregular star poly(3-hexylthiophene) via externally initiated Kumada catalyst-transfer polycondensation. ACS Macro Lett. 1, 392-395 (2012).

22. Ponomarenko, S. A., Kirchmeyer, S., Elschner, A., Huisman, B. H., Karbach, A. \& Drechsler, D. Star-shaped oligothiophenes for solution-processible organic field-effect transistors. Adv. Funct. Mater. 13, 591-596 (2003).

23. Kanibolotsky, A. L., Perepichka, I. F. \& Skabara, P. J. Star-shaped $\pi$ conjugated oligomers and their applications in organic electronics and photonics. Chem. Soc. Rev. 39, 2695-2728 (2010).

24. Senkovskyy, V., Beryozkina, T., Bocharova, V., Tkachov, R., Komber, H., Lederer, A., Stamm, M., Severin, N., Rabe, J. P. \& Kiriy, A. A core-first preparation of poly(3-alkylthiophene) stars. Macromol. Symp. 291-292, 17-25 (2010).

25. Van Den Eede, M. P., De Winter, J., Gerbaux, P., Teyssandier, J., De Feyter, S., Van Goethem, C., Vankelecom, I. F. J. \& Koeckelberghs, G. Controlled Synthesis and Supramolecular Organization of Conjugated Star-Shaped Polymers. Macromolecules 51, 8689-8697 (2018).

26. Steverlynck, J., De Winter, J., Gerbaux, P., Lazzaroni, R., Leclère, P. \& Koeckelberghs, G. Influence of the Grafting Density on the Self-Assembly in 
Poly(phenyleneethynylene)-g-poly(3-hexylthiophene) Graft Copolymers. Macromolecules 48, 8789-8796 (2015).

30. Shettigar, S., Umesh, G., Chandrasekharan, K., Rai, G. \& Kalluraya, B. Thirdorder nonlinear optical properties and two-photon absorption in polymers doped with p-phenyl Sydnone. J. Appl. Polym. Sci. 108, 3218-3224 (2008).

31. Boyd, R. Nonlinear optics. (Academic Press, 2008).

32. Gieseking, R. L., Mukhopadhyay, S., Risko, C., Marder, S. R. \& Brédas, J. L. applications: Guidance from theoretical and computational studies. Adv. Mater. 26, 68-84 (2014).

33. Chai, Z., Hu, X., Wang, F., Niu, X., Xie, J. \& Gong, Q. Ultrafast All-Optical Switching. Adv. Opt. Mater. 5, 1-21 (2017).

Hales, J. M., Barlow, S., Kim, H., Mukhopadhyay, S., Brédas, J. L., Perry, J. W. \& Marder, S. R. Design of organic chromophores for all-optical signal processing applications. Chem. Mater. 26, 549-560 (2014).

35. Sharma, S. K., Rezan, G. S. A., Misra, V. N. \& Tripathi, K. N. Third order nonlinear polymer materials for photonics. J. Mater. Sci. Lett. 22, 737-738 (2003).

36. Ma, H., Jen, A. K. \& Dalton, L. R. Polymer-Based Optical Waveguides : Materials, Processing, and Devices. Adv. Mater. 14, 1339-1365 (2002).

37. Van Steerteghem, N., Clays, K., Verbiest, T. \& Van Cleuvenbergen, S. ThirdHarmonic Scattering for Fast and Sensitive Screening of the Second 
Hyperpolarizability in Solution. Anal. Chem. 89, 2964-2971 (2017).

38. Moris, M., Van Den Eede, M. P., Koeckelberghs, G., Deschaume, O., Bartic, C., Van Cleuvenbergen, S., Clays, K. \& Verbiest, T. Harmonic light scattering study reveals structured clusters upon the supramolecular aggregation of regioregular poly(3-alkylthiophene). Commun. Chem. 2, 1-9 (2019).

39. Yokoyama, A., Miyakoshi, R. \& Yokozawa, T. Chain-Growth Polymerization for Poly(3-hexylthiophene) with a Defined Molecular Weight and a Low Polydispersity. Macromolecules 37, 1169-1171 (2004).

40. Sheina, E. E., Liu, J., Iovu, M. C., Laird, D. W. \& McCullough, R. D. Chain growth mechanism for the regioregular nickel inititated cross-coupling polymerisations. Macromolecules 37, 3526-3528 (2004).

41. Verswyvel, M., Monnaie, F., Koeckelberghs, G. AB block copoly(3alkylthiophenes): Synthesis and chiroptical behavior. Macromolecules 44, 9489-9498 (2011).

42. Rodriguez, V. Polarization-Resolved Third-Harmonic Scattering in Liquids. $J$. Phys. Chem. C 121, 8510-8514 (2017).

43. Ford, J. S. \& Andrews, D. L. Molecular Tensor Analysis of Third-Harmonic Scattering in Liquids. J. Phys. Chem. A 122, 563-573 (2018).

44. Spano, F. C. Modeling disorder in polymer aggregates: The optical spectroscopy of regioregular poly(3-hexylthiophene) thin films. J. Chem. Phys. 122, (2005).

45. Spano, F. C. \& Silva, C. H- and J-Aggregate Behavior in Polymeric Semiconductors. Annu. Rev. Phys. Chem. 65, 477-500 (2014).

46. Vertommen, S., Battaglini E., Elisabetta, S., Deschaume, O., Bartic, C., Verbiest, T. \& Koeckelberghs, G. The importance of excellent $\pi-\pi$ interactions in poly(thiophene)s to reach a high third-order nonlinear optical response. $J$. Phys. Chem. B 124, 9668-9679 (2020).

47. Terenziani, F., Katan, C., Badaeva, E., Tretiak, S., Blanchard-Desce, M. Enhanced two-photon absorption of organic chromophores: theoretical and experimental assessments. Adv. Mater. 20, 4611-4678 (2008). 